\title{
Design and Implementation of Double-Transmitter-Coil Antenna Used for the Tag Test System
}

\author{
Bin You, ${ }^{1}$ Haoling Yue, ${ }^{1}$ Xuan Wen, ${ }^{2}$ and Liangyu $Q u^{3}$ \\ ${ }^{1}$ The Key Laboratory of RF Circuits and System of Ministry of Education, Hangzhou Dianzi University, Hangzhou 310018, China \\ ${ }^{2}$ Nokia Siemens Networks Technology (Beijing) Co., Ltd., Hangzhou 310053, China \\ ${ }^{3}$ Shanghai TYD Electronic Technology Co., Ltd., Shanghai 200233, China
}

Correspondence should be addressed to Haoling Yue; yuehaoling@gmail.com

Received 30 August 2013; Accepted 22 September 2013

Academic Editor: Yu Jian Cheng

Copyright (C) 2013 Bin You et al. This is an open access article distributed under the Creative Commons Attribution License, which permits unrestricted use, distribution, and reproduction in any medium, provided the original work is properly cited.

\begin{abstract}
At present, more and more electronic tags are used in Antitheft system; it is important to examine the performance of electronic tags. The traditional single-transmitter-coil antenna (STC antenna) of the electronic tags tester has some serious drawbacks. So a novel double-transmitter-coil antenna (DTC antenna) is presented in this paper. Compared to the traditional STC antenna, this new antenna has a more excellent performance in the tag test systems, especially when it is used for testing the quality factor of tags.
\end{abstract}

\section{Introduction}

As the promotion of RFID and the widespread use of Antitheft system, the quality of electronic tags has gained more and more attentions [1]. But most of the tag detection systems cannot get the tag parameters accurately, especially the quality factor. Meanwhile, the detecting antenna plays an important role in the tag detection systems. So the research of detecting antenna has a great significance [2]. This paper designs a new DTC antenna which can perfectly solve the problems existed in traditional STC antenna and can be better applied to the tag detection systems. Besides, the DTC antenna can be also used for estimating Veff of tags [3].

\section{The Design of Antennas}

The frequency characteristic of tags cannot be measured directly. A test method based on the electromagnetic induction is often adopted. According to electromagnetic induction, two different coils are usually used as the antenna in the tag test systems $[4,5]$. One of these two coils is used as the transmitter-coil which sends the swept-frequency signal needed; the other one is used as the receiver-coil which tests the change of electromagnetic field around in real time. In this paper, this antenna is named as STC antenna. The electromagnetic field will change when there exists the electromagnetic induction between tags and the transmitter-coil. And then the change of electromagnetic field will be measured by the receiver-coil. Though simple, the STC antenna has some serious drawbacks. So this paper proposes a novel DTC antenna. This DTC antenna comprises two transmitter-coils and one receiver-coil and can perfectly solve the problems existed in STC antenna.

2.1. The STC Antenna. STC antenna has been widely used in tag test systems for a long time. The equivalent circuit of the STC antenna and the tag is shown in Figure 1. In Figure 1, $L 1$ represents the transmitter-coil of the STC antenna, LC resonance circuit represents the tag, and $L 3$ represents the receiver-coil of the STC antenna.

In order to simplify analysis, we assume that the current in receiver-coil is zero. According to electromagnetic induction, there is no electromagnetic field inducted by the receiver-coil. In other words, the receiver coil only can test the change of electromagnetic field around, but it will not interfere with the tag and transmitter-coil. Now, the current in transmitter-coil is $I_{1}$, and the current in the tag is $I$. So according to $K V L$, there are two equations (1) and (2) below 

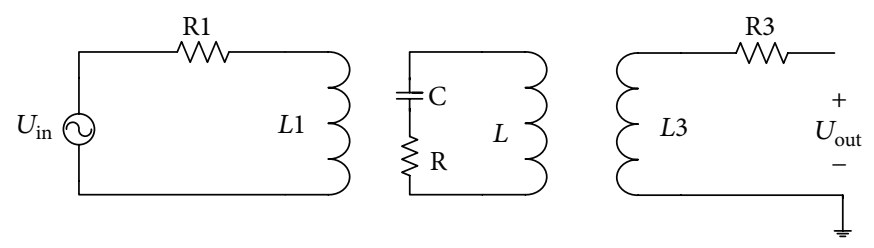

Figure 1: The equivalent circuit of the STC antenna and the tag.

where $Z_{1}, Z$, and $M_{1}$ represent the equivalent resistance of transmitter-coil, the equivalent resistance of tag, and the mutual inductance between transmitter-coil and the tag, respectively as follows:

$$
\begin{gathered}
U_{\text {in }}=I_{1} Z_{1}+j w M_{1} I, \\
0=I Z+j w M_{1} I_{1} .
\end{gathered}
$$

From the analysis of the equivalent circuit shown in Figure 1, the receiver-coil will be affected by both the tag and transmitter-coil simultaneously. Under the effect of the tag, the induced voltage output $U_{01}$ can be shown as follows:

$$
U_{01}=j w M_{3} I=\frac{w^{2} M_{1} M_{3}}{Z_{1} Z+w^{2} M_{1}^{2}} U_{\text {in }} .
$$

Similarly, the induced voltage output $U_{02}$ can be shown under the effect of the transmitter coil as follows:

$$
U_{02}=j w M_{13} I_{1}=\frac{j w M_{13} Z}{Z_{1} Z+w^{2} M_{1}^{2}} U_{\text {in }} .
$$

Finally, the total induced voltage output $U_{0}$ can be shown as follows:

$$
U_{0}=U_{01}+U_{02}=\frac{j w M_{13} Z+w^{2} M_{1} M_{3}}{Z_{1} Z+w^{2} M_{1}^{2}} U_{\text {in }} .
$$

The induced voltage measured by the receiver-coil is the sum of $U_{01}$ and $U_{02}$. Though the effect of transmitter-coil is relatively weak, it will finally affect the test result.

2.2. The DTC Antenna. From the analysis in Section 2.1, the STC antenna has two disadvantages. The first one is that the interference of tags with transmitter-coil may change the electromagnetic field distribution of transmitter-coil. The other one is that the interference of transmitter-coil with receiver-coil may finally change the test result. The design and implementation of DTC antenna are introduced in this paper. The most significant feature of the DTC antenna is that it can perfectly solve the mentioned problems existed in the STC antenna above.

The schematic diagram of DTC antenna is depicted in Figure 2. There is an operational amplifier in Figure 2. But the operational amplifier is not the essential part of the novel antenna. The effect of the operational amplifier is that it can make sure that the current in the receiver-coil is nearly zero because of its high input impedance. So According to electromagnetic induction, there is no electromagnetic field inducted by receiver-coil. In other words, the electromagnetic field inducted by tags will impact on receiver-coil, but not vice versa. Besides, the receiver-coil will not impact on the transmitter-coil. It is the premise of all next analysis. Moreover, the output voltage signal can be amplified by the operational amplifier so that it can be easily met the requirements of signal processing next.

The structure diagram of DTC antenna is shown in Figure 3. In Figure 3, the coil 1-1 and the coil 1-2 together make up the first transmitter-coil. Similarly, the coil 2-1 and the coil 2-2 constitute the second transmitter-coil. The two transmitter-coils must be placed symmetrically. In addition, the coil 3-1 and the coil 3-2 together make up a receiver-coil. Finally, two transmitter-coils and one receiver-coil make up the novel DTC antenna.

When the signal is sent to the two transmitter-coils, the directions of magnetic field inducted by the coil 1-1 and coil 1-2 are opposite. Thus, the magnetic flux of first transmitter-coil is zero; in the same way the magnetic flux of the second transmitter-coil is zero. Meanwhile, the magnetic field inducted by the coil 1-1 and coil 2-1 superimpose in one direction. In a word, the symmetrical structure of the two transmitter-coils insures that the receiver-coil will not interfere with the transmitter-coil.

So the inducted voltage of receiver-coil $U_{\text {out }}$ entirely depends on the tag. At last, there is an equation of $U_{\text {out }}$ shown below where the $M_{3}$ represents the mutual inductance between the receiver-coil and the tag and $I$ represents the current in the tag as follows:

$$
U_{\text {out }}=j \omega M_{3} I
$$

When tags are placed at the middle region of the coil 31 , the magnetic flux of the closed region shaped by the coil 1-1 and coil 2-1 or the closed region shaped by the coil 1-2 and 2-2 are all zero, no matter what the direction of magnetic field inducted by tags is. In other words, the magnetic field inducted by transmitter-coil can impact on tags, but not vice versa. Unlike the double layer structure of transmitter-coils, the receiver-coil has only one layer. Meanwhile, the magnetic field inducted by tags almost exists in the middle area of the coil 3-1. As a result, the coil 3-2 has been less affected by the magnetic field inducted by tags than the coil 3-1. So the change of magnetic flux of the receiver-coil is only dependent on the tag. The DTC antenna makes sure that there is no interference of the tag with transmitter-coils. 


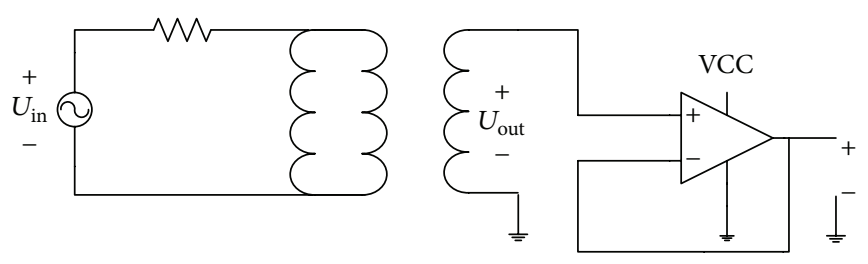

FIGURE 2: The schematic diagram of DTC antenna.

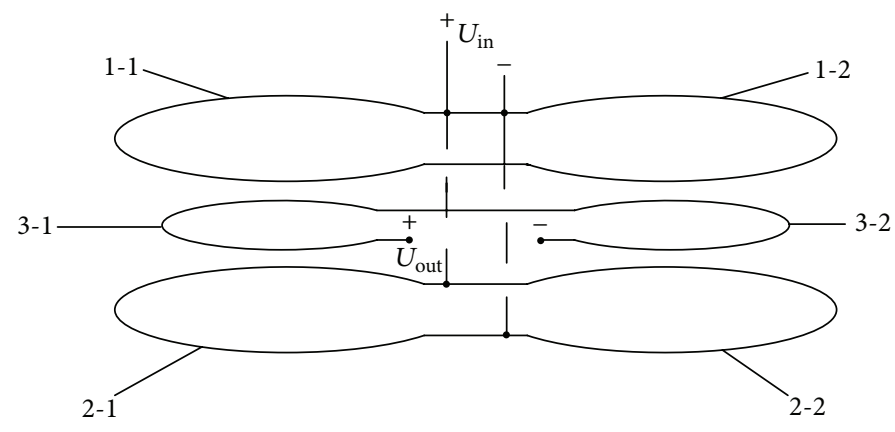

FIgURE 3: The structure diagram of DTC antenna.

So there are two equations as follows:

$$
\begin{gathered}
U_{\text {in }}=I_{1} Z_{1}, \\
0=I Z+j \omega M_{1} I_{1} .
\end{gathered}
$$

By synthesizing (6), (7), and (8), we can get

$$
U_{\text {out }}=\left(\frac{\omega^{2} M_{1} M_{3}}{Z_{1} Z}\right) U_{\text {in }} .
$$

From (9), the relationship between $U_{\text {out }}$ and frequency can be clearly found. The amplitudes of $U_{\text {in }}, M_{1}, M_{3}, Z_{1}$, and $Z$ in (9) are all constants for a given antenna. So the output voltage $U_{\text {out }}$ only changes with frequency and can reflect the impedance characteristic of the tag.

\section{The Hardware Implementation and Test of the DTC Antenna}

3.1. The Hardware Design of the DTC Antenna. According to the theoretical analysis above, the DTC antenna comprises two transmitter-coils which should overlap each other closely and a receiver-coil which should be placed between the two transmitter-coils. So the double-layers PCB as shown in Figure 4 is designed.

The two transmitter-coils are placed on the top layer and the bottom layer, respectively. The receiver-coil is placed on the top layer. Moreover, both the two transmitter-coils and the receiver-coil have the dumbbell-shaped structure. There are four via holes on the PCB; two of them on the middle of the dumbbell-shaped structure of the two transmitter-coils are used to import signals, and the rest two on the middle of the dumbbell-shaped structure of the receiver-coil are used to export signals. Furthermore, no matter which side of

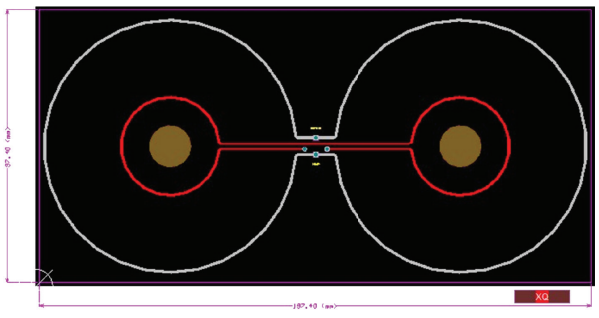

Figure 4: The PCB layout design of DTC antenna.

the dumbbell-shaped of the receiver-coil the tag are placed on, the test result will be the same.

In order to compare the performance of the two antennas, STC antenna is also designed on a single layer PCB shown in Figure 5. The concentric circle structure is adopted in the design. The bigger circle with two via holes stands for the transmitter-coil, and the smaller one represents the receivercoil.

3.2. The Performance Test of Two Antennas. A vector network analyzer E5071C, two antennas above, and some tags with known parameters such as the quality factor and center frequency are used in the test. The operational amplifier is not the essential part of the novel antenna. But in the practical application, in order to make sure the current in receiver-coil is zero, the output signal must be inputted into a signal amplification circuit. In the test, we use AD8067 as our operational amplifier. The complete testing circuit of the STC antenna is presented in Figure 6, and the testing circuit of the DTC antenna is presented in Figure 7. In the complete test, Port1 of E5071C supplies the swept-frequency signal needed to the transmitter-coil of the antenna. When the tags are placed on the antenna, the signal tested by the receiver-coil 


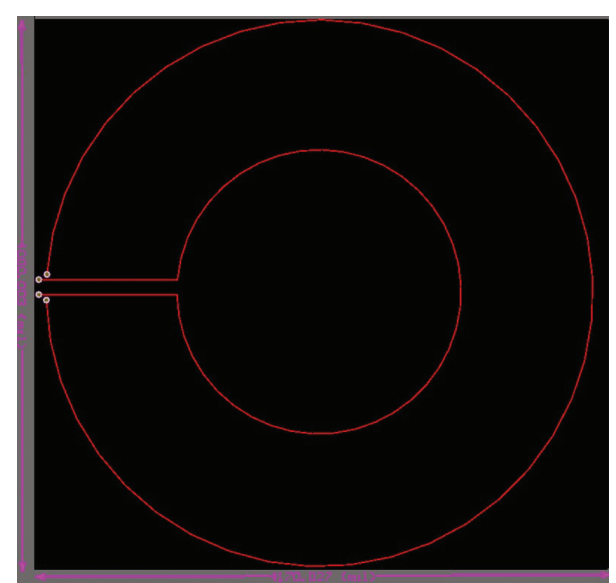

Figure 5: The PCB layout design of STC antenna.

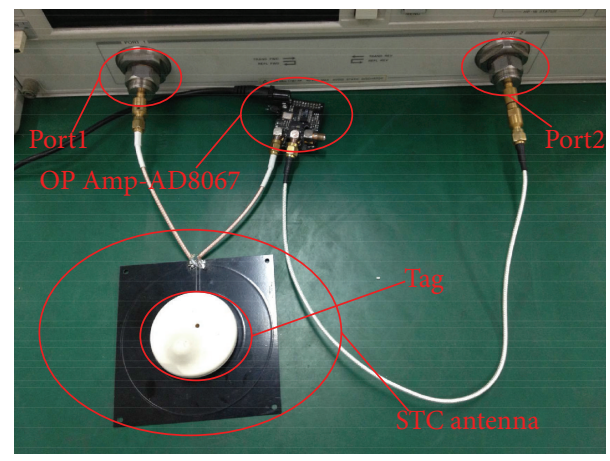

FIGURE 6: The complete testing circuit of the STC antenna.

TABLE 1: The test result of resonant frequency.

\begin{tabular}{lccc}
\hline & $f$ & $f_{1}$ & $f_{2}$ \\
\hline Tag 1 & $8.179 \mathrm{MHz}$ & $8.162 \mathrm{MHz}$ & $8.175 \mathrm{MHz}$ \\
Tag 2 & $8.053 \mathrm{MHz}$ & $8.000 \mathrm{MHz}$ & $8.033 \mathrm{MHz}$ \\
Tag 3 & $4.590 \mathrm{MHz}$ & $4.680 \mathrm{MHz}$ & $4.610 \mathrm{MHz}$ \\
Tag 4 & $4.759 \mathrm{MHz}$ & $4.880 \mathrm{MHz}$ & $4.809 \mathrm{MHz}$ \\
Tag 5 & $8.037 \mathrm{MHz}$ & $7.990 \mathrm{MHz}$ & $8.020 \mathrm{MHz}$ \\
\hline
\end{tabular}

is outputted to the input end of AD8067. The signal amplified by AD8067 is finally outputted to Port2 of E5071C so that we can clearly see the curves below.

When the tag with the center frequency $8.179 \mathrm{MHz}$ and the quality factor 151.1 is tested, there are test results as shown in Figures 8 and 9.

The test result of the STC antenna is shown in Figure 8.

It can be seen from Figure 8 that the test center frequency is $8.162 \mathrm{MHz}$ and the test quality factor is 125.5 .

From Figure 9, it can be seen that the test center frequency is $8.175 \mathrm{MHz}$ and the test quality factor is 151.3 .

Compared to two test results, the amplitude-versusfrequency curve measured by DTC antenna is more similar to the frequency characteristic curve of $L C$ resonance circuit. At the same time, the center frequency and the quality factor of tags measured by DTC antenna are more close to the real

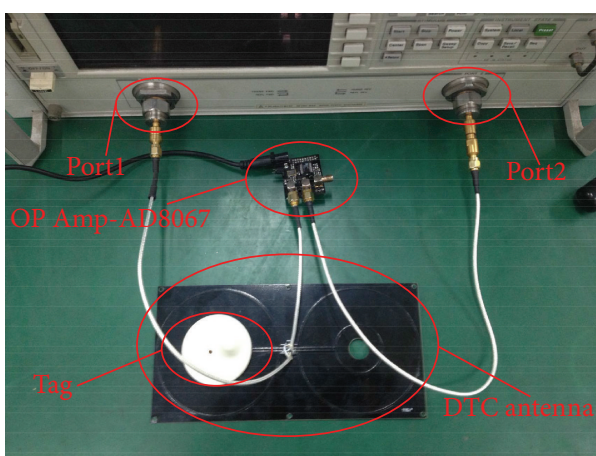

FIgURE 7: The complete testing circuit of the DTC antenna.

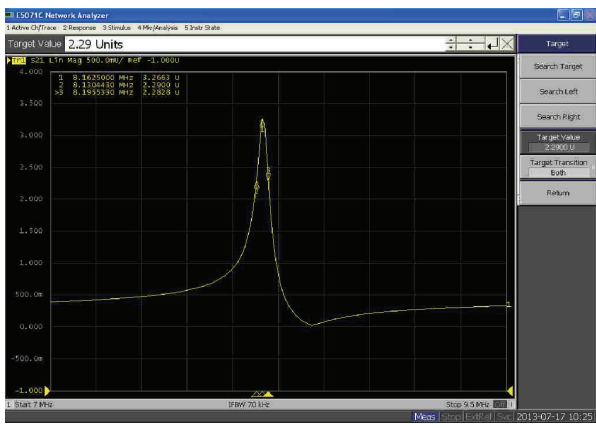

FIGURE 8: The test result of STC antenna.

TABLE 2: The test result of quality factor.

\begin{tabular}{lccc}
\hline & $Q$ & $Q_{1}$ & $Q_{2}$ \\
\hline Tag 1 & 151.1 & 125.5 & 151.3 \\
Tag 2 & 45 & 36.5 & 47.8 \\
Tag 3 & 96.9 & 55.1 & 102.4 \\
Tag 4 & 109 & 54.2 & 106.9 \\
Tag 5 & 49.1 & 36.3 & 48.3 \\
\hline
\end{tabular}

values. Compared to the two curves in Figures 8 and 9, there exists an unavoidable interference in STC antenna. The unavoidable interference of transmitter-coil with receivercoil finally leads to the difference between the two measured curves.

To proof the advantage of DTC antenna adequately, some kinds of tags are tested, and the test results are shown in Tables 1 and 2.

As shown in Table 1 , the $f$ is the real center frequency of tags. The $f_{1}, f_{2}$ present the center frequency of tags measured by STC antenna and DTC antenna, respectively. The $Q$ in Table 2 is the real quality factor of tags, while $Q_{1}, Q_{2}$ present the quality factor of tags measured by STC antenna and DTC antenna, respectively.

According to Table 1, the two measured center frequency are both close to the real values. But the measured curve of DTC antenna is more similar to the frequency characteristic curve of $L C$ resonance circuit. From Table 2, it can be clearly known that the quality factor measured by DTC antenna is 


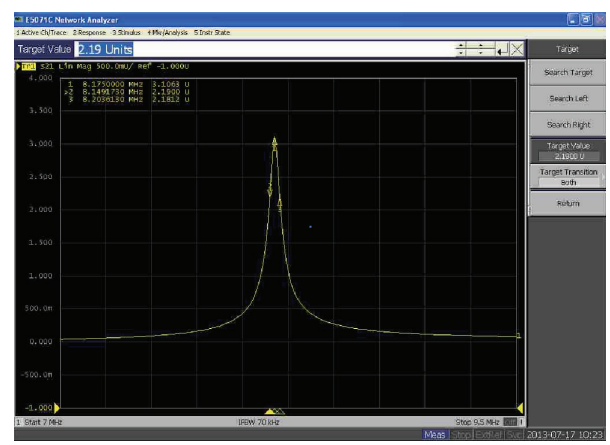

FIGURE 9: The test result of DTC antenna.

more close to the real value. To sum up, the performance of DTC antenna is better than STC antenna.

\section{Conclusions}

In this paper, a novel DTC antenna is proposed and completed. Experiments by DTC antenna have shown good capability in testing the quality factor and the center frequency of tags. So such an antenna model is more suitable for the tag detection systems than the STC antenna, especially the systems for obtaining the quality factor of tags.

\section{Conflict of Interests}

The authors declare that there is no conflict of interests regarding the publication of this paper.

\section{Acknowledgments}

This work was supported in part by the Zhejiang Province Key Science and Technology Innovation Team (no. 2010R50010) and the Real-time Antitheft system based on RFID technology (no. ZX130702307001).

\section{References}

[1] R. Want, "An introduction to RFID technology," IEEE Pervasive Computing, vol. 5, no. 1, pp. 25-33, 2006.

[2] Z. Ya-Ping, Z. Wei-Hong, X. Wei-Hua, and Y. Cheng-Zhong, "Development of EAS RF tag quality online detection sensor," Journal of Zhejiang University (Engineering Science), vol. 46, no. 4, pp. 719-724, 2012.

[3] W. B. Zou, Y. Wu, and Y. Zhao, "Automatic testing system for UHF passive RFID tag performance," in Proceedings of the 2009 International Conference on Networking and Digital Society, ICNDS 2009, pp. 79-82, Guiyang, China, May 2009.

[4] L. Jia-Jun, Y. Cheng-Zhong, Y. Zhi-Kai, and H. Lin, "The value of Q stability analysis by electronic anti-theft labels detection system," Journal of Hangzhou Dianzi University, vol. 32, no. 4, pp. 148-150, 2012.

[5] Z. Yanbin and L. Qin, "Design and realization of pulsed electronic article surveillance system," IEEE Intelligent Computation Technology and Automation, vol. 31, no. 2, pp. 28-29, 2011. 

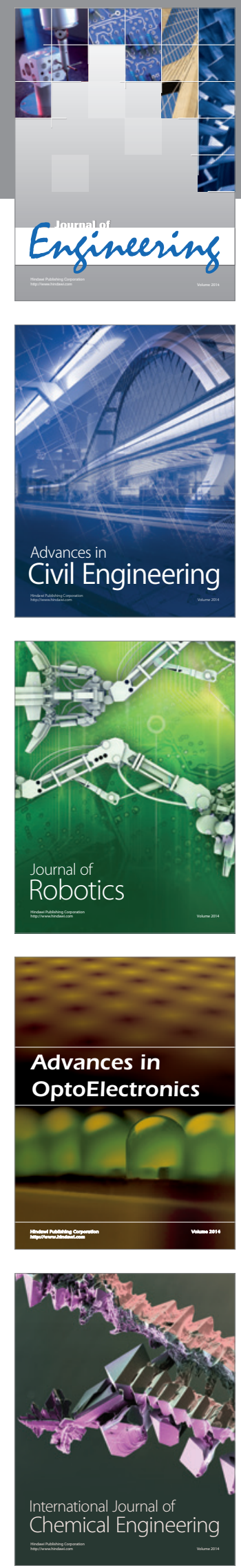

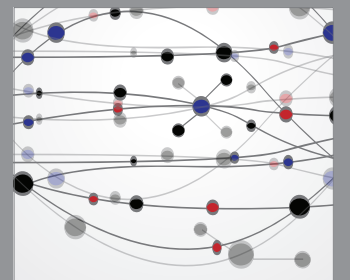

The Scientific World Journal
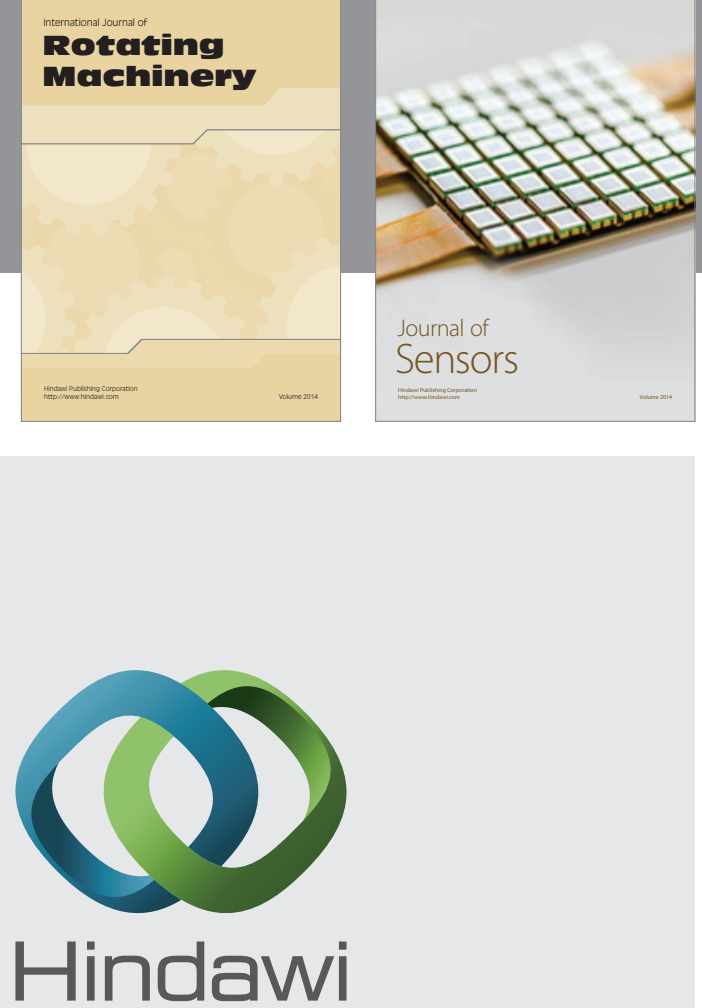

Submit your manuscripts at http://www.hindawi.com
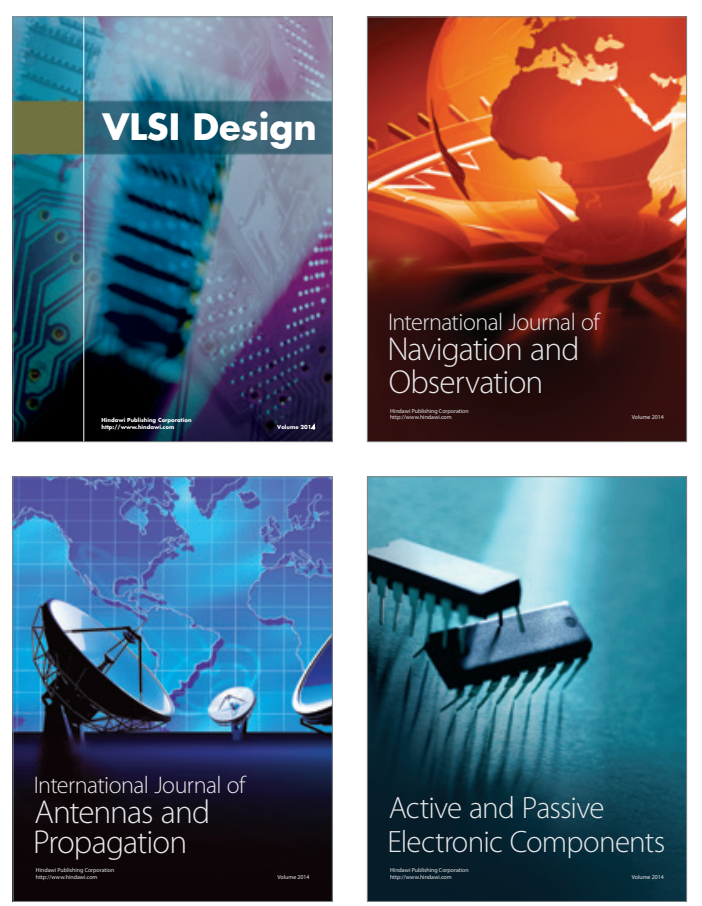
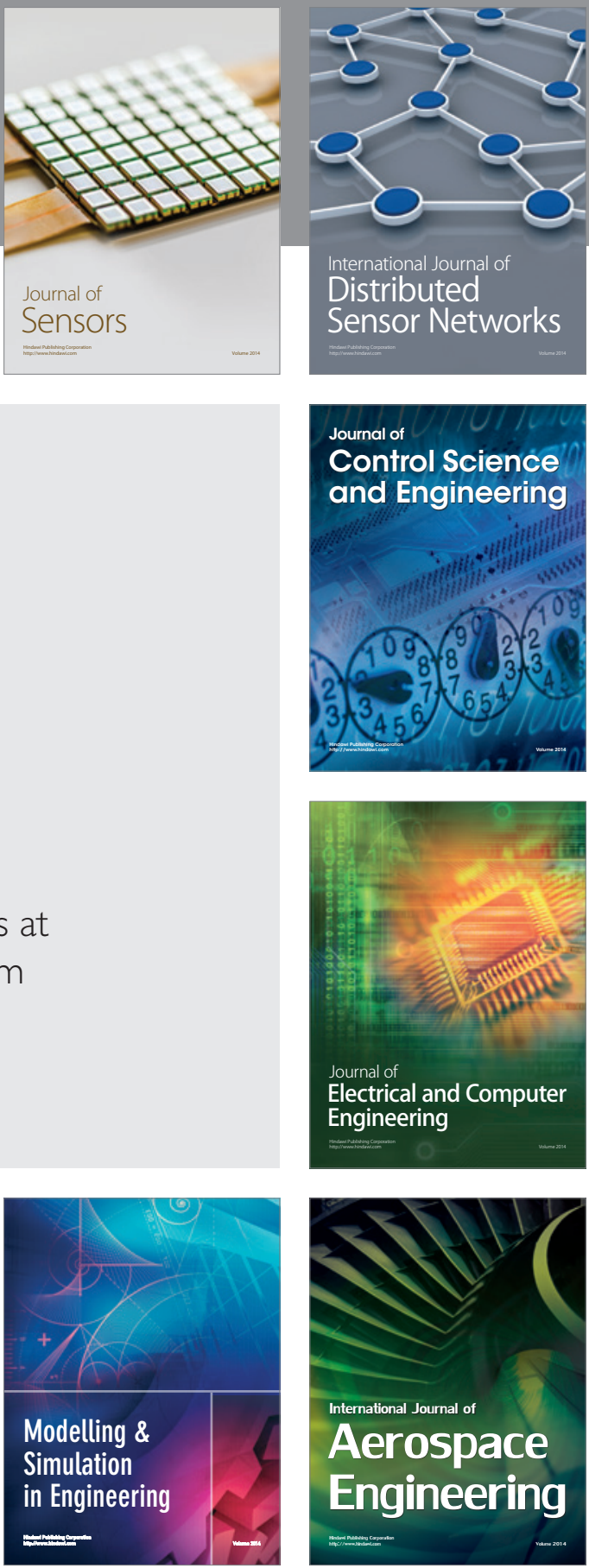

Journal of

Control Science

and Engineering
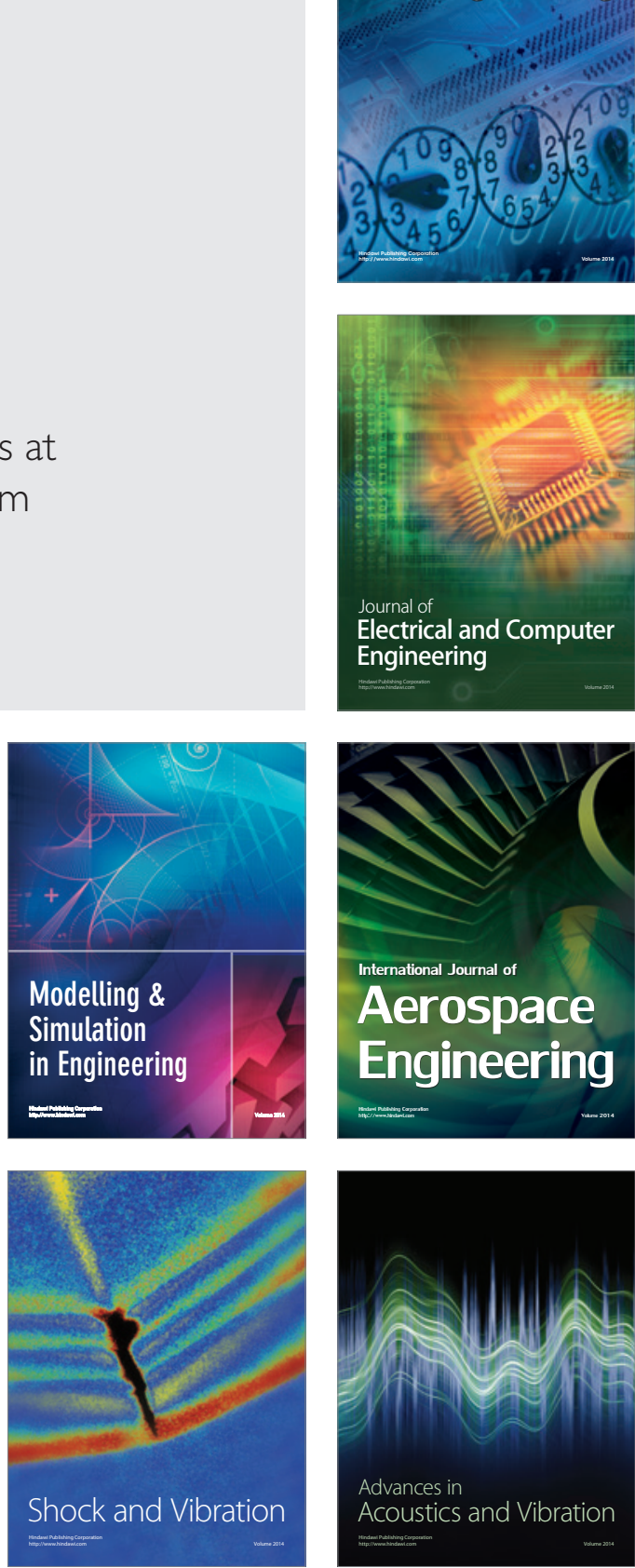